\title{
A Trauma Patient Advocate Is a Valuable Addition to the Multidisciplinary Trauma Team: A Process Improvement Project
}

Hartwell, Jennifer; Albanese, Kelly; Retterer, Abby; Martin, Stacey; O’Mara, M. Shay

The Joint Commission has identified communication as a common root cause of sentinel events. ${ }^{1}$ Trauma patients, in particular, report issues in communication with their care team and being confused about the treatment plan, prognosis, and options. ${ }^{2}$ Poly trauma patients who may be cared for by dozens of team members can easily get overwhelmed. Much has been written in the cancer literature about the role of a patient navigator to enhance patient comprehension of the disease, care coordination, follow up and improved communication. Results of patient navigator studies reveal that patients assisted by a navigator, or advocate, enjoy more efficient care, are more compliant with their care, and have lower mortality. ${ }^{3,4}$ To our knowledge, there is no description in the literature of a dedicated Trauma Patient Advocate (TPA) who's role it is to help trauma patients understand the complexity of their care, assist with team communication and trouble shoot clinical care and personal issues as they arise. Using a similar model of patient navigation, including the use of a multidisciplinary team and a dedicated TPA, we hypothesized that the addition of the TPA, would fill this void and would be perceived as effective by the clinical care team.

Grant Medical Center is an urban Level 1 Trauma Center that admits over 5000 patients annually. In July 2015, a process improvement (PI) project was started in which a dedicated Trauma Patient Advocate (TPA), known as A.R., was added to the multidisciplinary trauma team (MDTT) to assist patients and families through the complexity of trauma care. The TPA, a social worker by training and a hospital employee through the Department of Social Work, reported directly to one trauma surgeon champion informally each week, and to 
her service line administrator. The TPA attended the daily MDTT sign out conference. The MDTT consists of trauma surgeons, residents, nurse practitioners, case managers, social workers, charge nurses, a chaplain, a pharmacist, representatives from orthopedics, neurosurgery and plastic surgery, trauma registry staff, and program staff. Any member of the MDTT could request a consultation and assistance from the TPA on an individual basis after identifying specific patient needs on rounds and meeting the criteria of one or more of the following: patients with complex care issues such as poly trauma, having multiple consultants or service lines involved in the care of the patient, difficult family situations such as dealing with estranged family, difficult family communication due to work and travel schedules, need for assistance in decision making for discharge planning such as choosing a skilled nursing facility, or need for improved communication and coordination of medical care such as coordinating schedules for combined service line cases. After a 90-day pilot, a web- based survey was sent to the members of the MDTT to assess their perceptions of the TPA and her integration into the service. The administration and use of PI related survey results was approved by the Institutional Review Board of OhioHealth. Using a Likert-type scale, multiple choice questions and open-ended questions, respondents' answers were tallied.

During the trial period, the TPA saw 64 patients in 217 patient encounters. During interval updates from the TPA to the physician leader, the TPA reported the activities as listed in Table 1, which are grouped into five main categories: Service line communication, family information, trauma process, patient/family support, and customer service.

A web-based survey was sent to 365 members of the MDTT to assess the perceptions of the TPA's role, integration into the team, and effectiveness. Sixty-five MDTT members completed the survey for a response rate of $18 \%$. The results of the survey are summarized in Table 2. 
This report uniquely identifies the benefits of the TPA to the MDTT, not just for patients and families, but for the team members themselves. Team members reported improved patient care due to better communication, efficiency of information gathering and conflict resolution. In this PI project, we demonstrated that the dedicated efforts of one team member to help resolve customer satisfaction issues and provide education and support to patients and families was both well integrated into and well received by the MDTT. Team members correctly identified the TPA's role and had a strong belief that the work was valuable, as evidenced by the strong scores on the survey.

The free-text portion of the survey allowed team members to comment about their perceived relief when the TPA was able to spend time with "difficult" patients and families so the MDTT team member could continue his or her clinical duties and continue caring for other patients. While this was not the original goal of the TPA, we recognize now, the value of having the TPA free to address non-clinical issues, which are frequently time-consuming encounters. The TPA was perceived as a valuable resource to staff dealing with the "difficult patient/family" as she was able to devote uninterrupted time to patient/family support and assist with gathering and disseminating information about the trauma process, the current care plan, and even discharge planning. The coordination of these efforts is tedious and non-reimbursable. When the TPA was able to complete these tasks in parallel to the care team, there was a degree of perceived increased efficiency on the entire team.

The PI project was not intended to measure patient outcomes or satisfaction, but rather to fill a perceived void in the communication and coordination of care that our patients experienced on our busy urban trauma service. We were pleasantly surprised by the genuine passion our team members felt about the role of our TPA in the free-text portion of the survey. 
While it is hard to quantify this work, we discovered that by having someone fill the role of the TPA, who is not burdened by other clinical duties or documentation and billing requirements, we created a certain "je ne sais quoi" on the service; an intangible feeling of team work and connectedness between patients, families, nursing, therapists, and other service lines.

We do recognize, however, that the success of the TPA program in our institution may not be readily translatable to other trauma programs. The enthusiasm and engagement of the TPA strongly influences the effectiveness of the role. A.R. was not new to our hospital system and was known to many of the staff and physicians through her role in another hospital wide program. Her rapport with staff was already established and, no doubt, contributed to the success of her work as our TPA. The personality of the TPA plays a significant part in the quality of the program. A warm, engaging, and calm demeanor are essential characteristics of an effective TPA. The role is more relational than task oriented and securing the "right person for the job" is mandatory for success.

Additionally, we acknowledge that the response rate of $18 \%$ to our survey is low and there is inherent bias to positive feedback from those MDTT members who took the time to answer the survey and leave free-text comments. We recognize that generalizing the sentiments of $18 \%$ of the MDTT to the entire group is not appropriate. Though given the overwhelmingly positive statements in the free-text section and the very high overall scores, we feel confident in stating that there is enthusiasm for the TPA program and we will advance this pilot program into a permanent role on our MDTT.

In conclusion, we determined that the addition of the TPA to our busy, urban Level 1 trauma service was well received by the MDTT, subjectively improved team communication 
and efficiency, and was perceived to increase patient and family engagement and satisfaction with the trauma experience.

\section{REFERENCES:}

1. http://www.jointcommission.org/assets/1/18/Root Causes by Event Type 20042014.pdf; last accessed 10/2/15

2. Gabbe BJ, Sleney JS, Gosling CM, Wilson K, Hart MJ, Sutherland AM, Christie N; Patient perspectives of care in a regionalized trauma system: lessons from the Victorian State Trauma System. MJA 2013; 198 (3): 149-152.

3. Freeman HP, Muth BJ, Kerner JF. Expanding access to cancer screening and clinical follow-up among the medically underserved. Cancer Pract 1995; 3(1): 19-30.

4. Noel L, Connors SK, Goodman MS, Gehlert S. Improving breast cancer services for African-American women living in St. Louis. Breast Cancer Res Treat 2015; 26 Sept; open access at Springerlink.com; last accessed 10/2/15 
Table 1: Unique TPA Activities (per TPA Self-Report)

\begin{tabular}{|l|l|}
\hline Service Line Communication & $\begin{array}{l}\text { Aided in communication between trauma, orthopedics, } \\
\text { neurosurgery, and plastic surgery }\end{array}$ \\
\hline Family Information & $\begin{array}{l}\text { Assisted APP and physicians in relaying information to } \\
\text { patients' families when they were unavailable during }\end{array}$ \\
\hline rounds & $\begin{array}{l}\text { Assisted in securing information about OR times for } \\
\text { family members }\end{array}$ \\
\hline Trauma Process & $\begin{array}{l}\text { Arranged and attended family meetings to discuss goals } \\
\text { of care }\end{array}$ \\
\hline Assisted patients, families with the completion of \\
paperwork (FMLA, worker's comp, etc)
\end{tabular}


about their loved one's care

Table 2: The TPA Survey sent to the MDTT with results

A.R. has recently joined the trauma team. Her current work is a pilot program and we are soliciting ways to improve her interaction with patients and better define her role.

Please take a moment to answer the following questions.

1. Which of the following best describes A.R.'s role with the trauma team?
A. She is a new case manager
B. She is a new nurse
C. She is a new trauma patient advocate Response: $95 \%(62 / 65)$
D. She is a new therapist

2. Have you interacted with A.R. either in multidisciplinary morning check out conference or in direct patient care?
A. Yes Response: $57 \%(37 / 65)$
B. No

3. A.R.'s interaction with patients is valuable to the trauma team.

Agree $5 \quad 4 \quad 3 \quad 2$ 1 Disagree Response: 4.42

4. A.R.'s interaction with patients is valuable to the patients.

Agree $5 \quad 4 \quad 3 \quad 2$ 1 Disagree Response: 4.46

5. A.R.'s involvement improves the trauma patient experience.

Agree $5 \quad 4 \quad 3 \quad 2$ 1 Disagree Response: 4.37

6. I know how to get a hold of A.R. either directly, or through someone else.
A. Yes Response: $69 \%(45 / 65)$
B. No

7. Who can refer patients to A.R.?
A. Nursing staff
B. Trauma attendings, residents or NPs
C. Case managers/social workers
D. PT/OT/ST
E. Any of the above Response: $97 \%(63 / 65)$

8. Which of the following best describes A.R.'s goals in seeing trauma patients?
A. Trouble shoot communication issues
B. Resolve patient satisfaction issues
C. Address family issues 

D. Gather information from the treating team (nursing, therapy, physicians, etc) and help patients understand the trauma experience
E. All of the above Response: $95 \%(62 / 65)$

9. Which of the following patients would be best to refer to A.R.?

A. 45 year old male after a fall with an isolated left tib/fib fracture, he is doing well with therapy and is one day away from discharge. He lives in town and has good family support; they are usually at the bedside with him.

B. 27 year old male after a motorcycle accident who has a small SDH (brain injury), a left radius fracture, a left femur fracture, multiple facial fractures, and had an emergency ex lap (surgery) to remove his spleen. His family lives about 75 miles away and there is some confusion about whether it will be best for him to be discharged to home or to inpatient rehab. The family is intermittently at the bedside and is confused about the options. Response: 95\% (62/65)

C. 80 year old female after a fall who has no injuries; she is being monitored overnight because she is on aspirin and Plavix; the team is awaiting a geriatrics consult; she will return to her SNF

D. 33 year old male who was intoxicated, involved in an MVC; he has no documented injuries other than a concussion; he is awaiting a cognitive evaluation and will likely be discharged home later today

10. Describe an interaction you have had with A.R., either directly or that you have observed a patient have.

11. Describe how you envision A.R. expanding her role and becoming even more effective in the care of the trauma patient. 